\title{
The Impact of the Maternal Sepsis Intervention
}

\begin{abstract}
This chapter presents data on maternal mortality in Uganda and the contribution that sepsis makes to mortality. Against this backdrop, it identifies key outcomes of the intervention including major improvements in maternal mortality and reductions in the length of patient stays, readmission rates and hospital expenditure.
\end{abstract}

Keywords Antimicrobial resistance - Antimicrobial stewardship . Infection Prevention Control · Maternal mortality · Maternal sepsis

It is common in ODA projects to be required to produce 'outcome indicators' related specifically to 'beneficiaries'. As noted in the discussion on methods, the expectation is usually for quantitative metrics. The funding call and associated guidance, unusually for UKAid projects, did not specify patient outcome indicators but focused more on stewardship processes and antimicrobial consumption data. It is also more usual, in research, to discuss the intervention processes in more detail and report the findings at the end. The project proved to be more impactful than we had anticipated with significant reported impacts on maternal mortality, patient stays and hospital budgets. ${ }^{1}$ We have chosen to report these outcomes first and

\footnotetext{
${ }^{1}$ It may be interesting to note that these outcomes were not predicted or described in the MSI application or present in the logframe as we felt they would have seemed

(C) The Author(s) 2020

L. Ackers et al., Anti-Microbial Resistance in Global Perspective, https://doi.org/10.1007/978-3-030-62662-4_3
} 
then to try to unpack the processes contributing to these outcomes. As we noted in the methods chapter, funding bodies and researchers are as much concerned with attribution (and change processes) as they are outcomes. And in such complex interventions many variables will intervene to influence attribution and outcomes. In action research, some of these variables will come directly from iterative responses to ethnographic understandings of context; others will come from external, often hidden, processes.

\section{Maternal Deaths from Sepsis at Fort Portal Regional Referral Hospital (FPRRH)}

Allegranzi et al.'s systematic review (2011) reported the very poor quality of facility data on health-care-associated infections and associated impacts:

Unfortunately, in studies retrieved findings related to increased length of stay and attributable mortality and costs associated with health-careassociated infection were fragmentary. (2011: 236)

Qualitative interviews with health workers and stakeholders repeatedly referred to the fact that there had been no deaths from sepsis at FPRRH since the MSI became operational. Many of the respondents alluded directly to reductions in maternal deaths and the effects of this on the reputation of the wards:

FPRRH was identified once again as the RRH in Uganda with the highest maternal mortality rate. It was in the newspapers and the Director is very concerned and embarrassed about this so any improvements on the ward are very welcome. (midwife)

Health workers were keen to cite examples of patients whose lives had been saved because of (their) improved practice. They were not only delighted and highly motivated by their ability to save these women's lives, but the experience also provided direct affirmation of the value of behavioural changes on the wards:

overly ambitious had we been required to deliver on them. This process could lead to an under-statement of impacts in ODA projects. 
We have had 4 complicated cases (this week). We thought the patients would die and they walked out happy. There was a patient we thought would pass away, but she survived as she got the appropriate antibiotics. (midwife)

These responses, supported by our observations on the wards, encouraged us to undertake a review of maternal deaths. In practice, it proved difficult to find all the cases and to identify a clear causal factor in the files. After detailed analysis of the documentation, Fig. 3.1 documents a reduction in deaths from sepsis over time since implementation began in June 2019. We tracked the data back to 2018 to assess trends and this revealed some unexplained peaks and troughs.

Although most responding health workers referred to the sharp decline in deaths, analysis of the maternal mortality data indicated the persistence of deaths associated with sepsis. We could describe the method here as a forensic ethnography, and an example of where a mismatch in perceptions and data led us to delve deeper. The data support the contention that, since implementation, there have been no deaths from sepsis amongst those women having c-sections in FPRRH. One of the 'training' mechanisms the project team have instigated involves regular audit meetings on the wards; these include all cases of maternal death or serious sepsis and provide an opportunity to assess practice and monitor outcomes. Each of the sepsis deaths discussed in these meetings have involved women referred into the hospital from health facilities some distance away

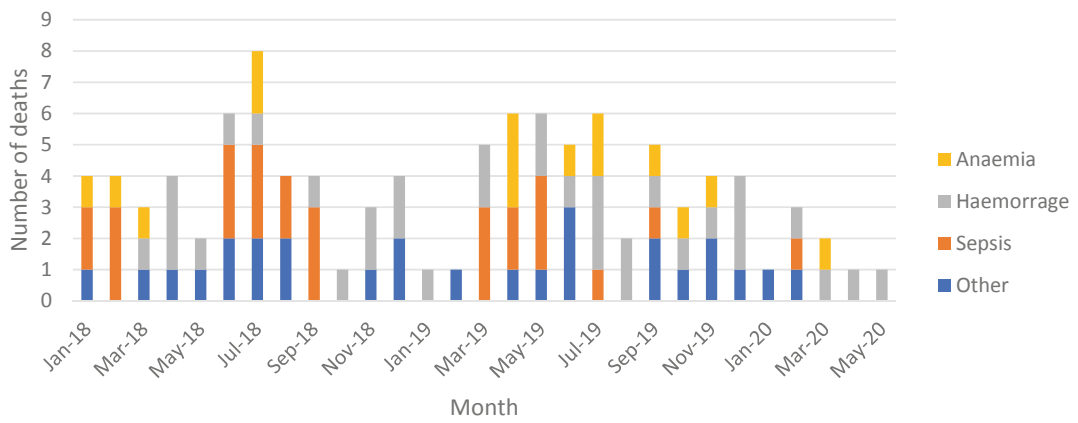

Fig. 3.1 Causes of maternal death (FPRRH) (Source Maternal Mortality Registers and Files, January 2018-May 2020) 
primarily in refugee camps. These women face major referral pathway delays and arrive at the hospital late at night often in a very critical condition.

\section{'The RoAd to DeATH'2: Understanding THE IMPACT OF GEOGRAPHY AND DELAYS}

Regional Referral Hospitals have a particular role to play in the Ugandan public health system. That role is predicated on the efficacy of the referral system as a whole and the functioning of lower level referral facilities so that only those cases that need treatment at a specialised facility should be referred there. This system is barely functioning. One of the major problems facing FPRRH is congestion caused by systems malfunction and significant delays in referrals with many women dying shortly after arrival. The only deaths from maternal sepsis during the project have involved late referrals from distant facilities and refugee camps. These cases either stemmed from or bypassed facilities with responsibility for Emergency Obstetric Care that should have been able to treat them in a timely and effective fashion. At the request of the hospital director, we undertook an analysis of the residence of women who die at FPRRH. This illustrates the distance many had to travel before reaching FPRRH.

Aware of the impacts of such deaths on the reputation of the hospital the Director encouraged the team to explore this issue in more detail. Analysis of a further 1000 admissions onto maternity evidenced similar referral patterns.

This data was not planned for in the application but provides critical context to an understanding of sepsis (and AMS) at the hospital. The interviews with health workers and stakeholders illustrate the problems associated with travelling long distances to access FPRRH. One intern doctor commented:

Our ward is very small, and the volume of patients is overwhelming because the nearby hospitals are not doing $\mathrm{c}$-sections. We have patients now coming from Kyenjojo hospital where they are not doing sections; they just put on the form that they have no anaesthetists.

${ }^{2}$ Filippi et al. (2005). 
Other respondents talked about the problems of having women referredin especially from Kyenjojo (50 km away) and Mubende (145 km away); facilities which have theatres and post-natal wards.

But why are the doctors not working there? We have heard it is because the anaesthetists are not on the ground. They are bumping the cases down here simply because their anaesthetists are not there. In the referring centres you will find zero deaths - they will keep a mother there until she is now risky and between leaving there and arriving here... most women die within $1 \mathrm{~h}$ of arriving. (stakeholder)

Another (stakeholder) respondent referred the importance of distinguishing those sepsis cases arising from local operations and those coming in through referrals or, as is often the case, self-referrals:

Of the patients operated in Buhinga fewer are having sepsis. We should distinguish these from those coming from outside of Buhinga; we have 2 separate entry points.

Sadly, a sepsis-related death occurred in February 2020. The case illustrates the challenges facing a hospital when women face extensive delays. In this case, the woman travelled some $77 \mathrm{~km}$ from Rwamwanja Health Centre, near Kamwenge. This area supports a refugee camp. The case was reported to us as follows:

The 18-year-old woman was referred to FPRRH 6 days after an emergency C-section apparently for obstructed labour and a still birth. When she arrived late at night doctors removed a retained swab and started antibiotics. The following morning, she was found to have intra-abdominal sepsis and needed another operation to wash the pus out and remove her uterus which was the source of the infection. You could smell the infection from metres away. We took her to theatre, washed out her abdomen, and removed her uterus, which was severely infected and necrotic. We then put her on meropenem, our strongest possible antibiotic. She later died.

Causes of death: Poor monitoring of labour and delayed caesarean at the health centre, leading to foetal death and increased risk of infection. Likely poor asepsis and retained swab during the caesarean leading to postoperative infection. Delay in recognition that she was not getting better resulted in delays in referral to FPRRH. Possible antibiotic resistance meant that any initial empirical treatment was not enough and possibly there was 
even resistance to meropenem (though she might have been too far gone by the time she reached us).

Figure 3.2 shows that six of the maternal deaths in 2019 came from this refugee camp as well as a large volume of admissions (Fig. 3.3). Respondents have advised us that, although health facilities in such camps are typically better resourced and ring-fenced UNHCR funds are available for transport to FPRRH, drivers obtain greater funding for night visits and are therefore inclined to delay transportation of very sick patients until night-time. Other respondents suggested drivers may wait until they have 3 or 4 cases to transport, especially if any of those cases are local Ugandans who do not come with a budget. It has also been reported that where local Ugandan women do manage to get access to this transport to FPRRH they are not permitted to travel back via this route. Whatever the reasons, late referrals such as this one leave little opportunity for staff at FPRRH to save lives and this also directly impacts antimicrobial stewardship.

The impact of late referrals on maternal mortality from sepsis is confirmed by Ngonzi et al. (2016) in Uganda and Kongnyuy et al. (2009) in Malawi. Seni et al. (2013) put another twist on this relationship between late referrals and SSI incidence reporting an 'alarming' prevalence of SSI in Uganda amongst 'emergency surgeries which accounted

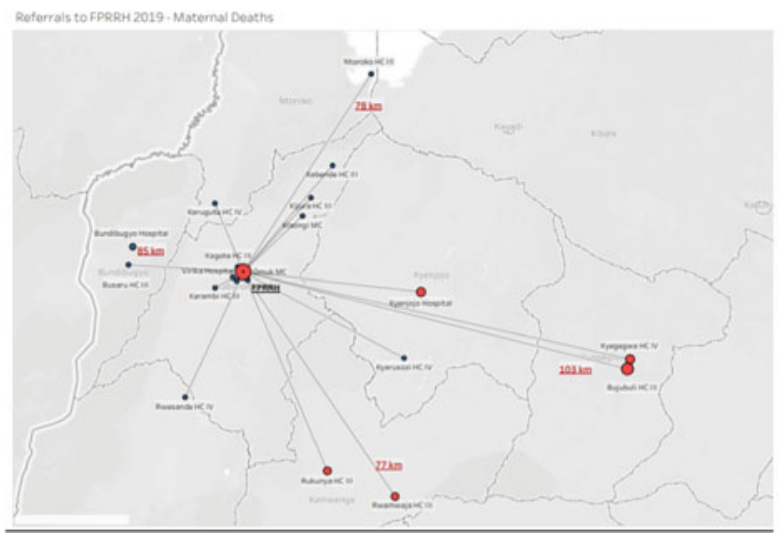

$\begin{array}{lr}\text { District } & \\ \text { name } & \text { Frequency } \\ \text { Bundibugyo } & 3 \\ \text { Bunyamurwa } & 1 \\ \text { Bunyangabu } & 3 \\ \text { Kabarole } & 15 \\ \text { Kamwenge } & 6 \\ \text { Kyegegwa } & 11 \\ \text { Kyenjojo } & 8 \\ \text { Ntoroko } & 2\end{array}$

Fig. 3.2 Distances travelled to FPRRH (maternal mortality cases) (Source Analysis of maternal mortality cases, 2019) 

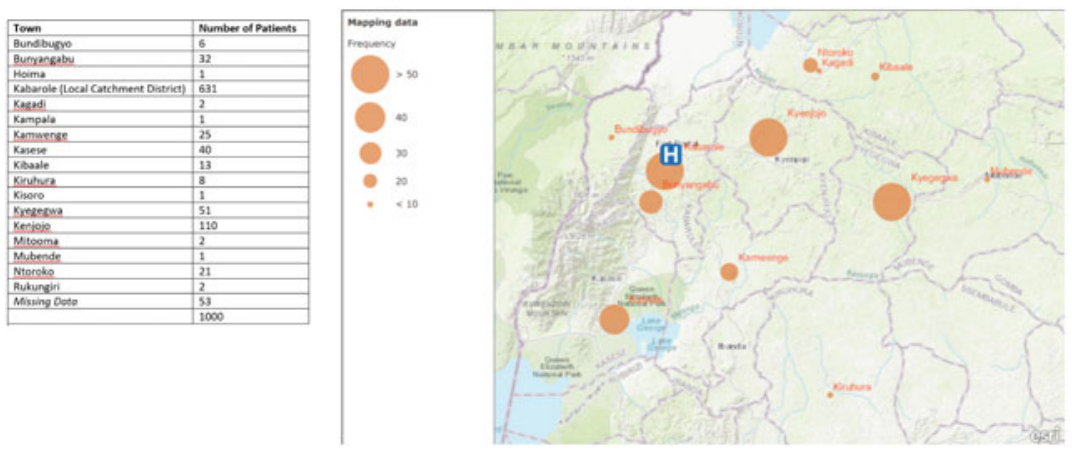

Fig. 3.3 Distances travelled to FPRRH (Admissions, 2019) (Source Analysis of 1000 Maternal Admissions, 2019)

for more SSIs cases as opposed to elective surgeries' (2013: 5). Very few Health facilities in Ugandan run elective list for non-fee-paying cases. All the referrals coming into FPRRH are for obstetric emergencies. It is easy to understand the hospital director's concerns about these cases and the attribution of blame. The relationship between adverse patient safety events and reputational damage is noted by Slawomirski et al. (2017) in a report on the economics of patient safety (2017). The authors cite the results of several studies in High Income Settings illustrating the impact of adverse patient safety events on prolonged hospital stays. They conclude:

Hospital-acquired sepsis accounts for a large proportion of additional hospital days, standing out as one of the most expensive and most clinically complex conditions to treat. (2017: 16)

The data presented above and the discussion about referral cases could be seen as a distraction from the main focus of the research. We believe it illustrates the importance of a very deep and intuitive approach to context. On the one hand, the ability to evidence the source of sepsis cases has supported local policy and underlined the need to invest in these referral facilities and, potentially, extend the intervention to such sites. On the other, the data and mapping process has served an important function in challenging the basis of media coverage of the hospital (and its position in maternal mortality league tables). This has proved a real motivation for hospital managers and health workers alike. 
One of the studies cited by Slawomirski et al. (2017) (above) reported patients staying 10.2 days longer in Dutch hospitals as a result of adverse patient safety events (Hoonhout et al. 2009). Seni et al. (2013) report the relationship between very high levels of SSI in Uganda with 'substantially longer hospital stays and higher treatment costs' (2013: 6). The following section evidences reductions in patient stays as a result of the MSI.

\section{Impacts on Patients, Their FAMILIES, AND LIVELIHOODS}

Spending time in hospital is a significant burden in low resource settings especially amongst the poorest of patients. During their time in hospital, they are unable to undertake any economic activity or caring responsibilities themselves and often have up to five or six attendants residing in or near the hospital to support them, providing food and routine care. Many patients admitted to a Regional Referral Facility will have travelled some distance from their home village. The large number of women described in the notes as 'running away' (or self-discharged) prior to completion of their treatment ${ }^{3}$ (and at high risk of readmission or mortality) do so in order to care for children and family and to enable their families to return to their activities. Extended stays in busy referral facilities also contribute directly to congestion. This presents a challenge in terms of maintaining adequate IPC. Congestion is often manifested in floor cases making it hard to clean facilities effectively and for over-stretched staff to deliver efficient and respectful care. The fact that every patient will have a number of attendants many of whom sleep and eat amongst the patients on the floors is a further hazard. Zulfiuqar et al. (2013) reported concerns about the security and infection risks posed by the presence of in-patient attendants at a tertiary hospital in Karachi. Most patients in their study had at least one attendant with $85 \%$ attendants staying for the duration of the patient stay and performing critical care duties for patients including feeding, washing and administering of medications. Extended stays of patients and accompanying attendants create the conditions for the spread of Healthcare Associated Infections some of which have shown multidrug resistance (Acinetobacter). Where extended stays are due to delays

\footnotetext{
${ }^{3}$ These cases contribute to the poor quality of antibiotic consumption data as we have no way of knowing if they complete their prescribed dose.
} 
in closing wounds (secondary closure), ${ }^{4}$ this also creates opportunities for secondary infections.

Most respondents expressed the view that patients were staying for shorter periods on the ward and they were experiencing fewer readmissions due to sepsis. The following midwife suggests that the project has had a significant impact on length of stay:

These days the patients do not stay on the ward for very long. At first those ones with sepsis they could spend 2 weeks or 4 weeks on the ward but now you find a mother has come with sepsis - you do the dressing a mother will not even spend a week on the ward. Because they remove a swab - they take it there - we get the results - the doctor comes and investigates and the good thing we are doing closure now in our room - we are not now taking them to theatre because thanks to $\mathrm{K} 4 \mathrm{C}$ we now have the instruments to do them there and to sterilise them.

This observation is backed up by the laboratory:

Patient stays have really reduced on the ward and we produced evidence on this last year. Patients were staying for over 30 days on the ward prior to the project - now in many cases after culture and sensitivity testing 5 and closing the wounds they can then go home after 10 days. We have reduced patient stays on the ward and even in terms of costs, shorter stays and fewer attendants on the wards.

One midwife explains how reductions in length of stay are a combined result of improved wound care, improved prescribing in response to laboratory results and the infrastructural improvements that have improved the functionality of the evacuation room. This involved the provision of additional instruments, sterilising equipment and storage cupboards and has enabled intern doctors to close (re-stitch) wounds on the ward

\footnotetext{
${ }^{4}$ Secondary closure is a term used on the ward to describe cases where the original c-section wound opens as a result of infection. In some cases, the staff have to re-open infected wounds. Some of the wounds on the ward gape openly with a diameter of up to $10 \mathrm{cms}$. They then must be debrided (removal of damaged tissue) and dressed until the infection is gone and they are able to re-close them.

${ }^{5}$ Culture and Sensitivity Testing is the term used to describe the process of taking a sample from a patient; growing the bacteria from that sample in a laboratory and then testing the susceptibility of (or responsiveness to) any bacterial growth to available antibiotics.
} 
without sending women to surgical theatre. Theatre days for elective wound closure are only available twice a week and if a woman misses that date, she may have to remain on the ward with an open wound for another 4 or more days. The savings in such cases include not only shorter stays but reduced use of precious theatre time. As one doctor put it, 'wound closures cannot be a priority for theatre time'. This also reduces the risks associated with moving patients around the hospital. An intern doctor describes the changes he has witnessed on the ward:

The instruments in the evacuation room means we can now do procedures that used to take a long time as we had to sterilise equipment between cases so mothers can be treated much more quickly, get better and leave the next day. This takes pressure off theatre too. We now dress (the wounds) and when they are ready, we do the secondary closures - they don't need to go to theatre. We have been able to move women out of the ward more quickly which has reduced congestion as women heal more quickly now. We do it in the evacuation room and now we disinfect it and we know we have to reach higher standards so we can close the wounds there. The doctors are doing most things now - the interns will do some without supervision apart from difficult wounds then they take to theatre and the senior doctors are there. They will assess the wounds and we do what we can here.

[Were the doctors doing this before?]

Actually, they were doing some closures, but it would take some time - they had to wait to disinfect the equipment but now it is streamlined. It has really improved now; the numbers of mothers that need to go to theatre has reduced a lot - because what happens - the mother comes with a bad wound - these guys do the dressing and manage the mothers and the wound becomes small and easy to suture so we can do it here now on the ward. The sepsis has really gone down but maybe out of 5 we can now take 2 to theatre and the other 3 we can close the wounds here. Before the number was big and all mothers were being taken to theatre to close the wounds. In fact, in the last 2 months we have had a situation where the septic mothers' section is empty - the place is clean - you have done a great job.

Another intern refers to improvements in patient recovery and length of stay:

What [MSI] has brought to reality is that - actually when samples are taken and followed up then things get better more quickly - as observed you will 
note that this side has very limited sepsis now - there are few and even those that are there are improving so quickly and we are doing secondary closure so quickly. Before we had patients on the ward with a wound for over a week and as you bring in a new patient, they get a hospital acquired infection but now hospital stay has been reduced from 2 weeks to 5 days... we really could quantify this. We now have fewer infected wounds to dress and fewer secondary closures and we can do many of these now on the ward. Before every infected patient was cross-infecting the next patient the longer they are on the ward the more this happens. We have outgrown waiting to go to theatre.

The doctor also refers to the benefits of having a new trolley and instruments but, importantly, the impact of leadership and improved human resource management:

Most of the secondary closures are being done on the ward now. Now we have the instruments and the trolley - before there wasn't anyone who would take responsibility to wash the instruments so there was a delay now the instruments are enough - the challenge was for someone to take responsibility for washing and sterilising them. Now we do the closures as soon as they are ready - before the room was not sterile for secondary closures but now it is ready. There are now few cases going to theatre unless it is more serious like a burst abdomen. Before in the evacuation room the instruments were lying in jik (disinfectant) so you had to wait. Then the patients can become septic again. Now you can't spend a day waiting.

Another midwife adds the observation that this has also reduced the volume of readmissions:

The wounds get better and then the interns can do the secondary closures and we have had no evidence of readmission of these cases.

When the project started, we were advised that many of the cases on the post-natal and gynae wards were re-admissions; that is women who had been treated on that ward and discharged and then had to return due to infection. In March 2020, the midwifery team decided to track every woman coming onto the ward with an infected wound for a period 
of 3 weeks. Of the 121 cases tracked, there was no evidence of readmission. ${ }^{6}$ These perceptions are backed up by the evidence drawn from facility records. In a pain staking process and with support from the hospital administrator, the team have worked with the records office to reorganise a mass of files into date order and entered data into a spreadsheet of every case of sepsis in 2019-2020. This has provided a rich database enabling us to assess, objectively, the impact of the intervention on patient stays.

\section{Impact on Patient Stays}

The following section reports on an analysis of all cases of suspected sepsis recorded over a 13-month period from January 2019 up to and including January 2020. 142 cases were identified in patient records which recorded patient stays from the date of operation (usually a c-section) to discharge. A t-test was done to determine whether there was a difference in hospital stays between those patients who had received a culture swab and those who had not. It was found that hospital stays were significantly lower in patients for whom culture swabs were completed $(\mathrm{M}=21.28, \mathrm{SD}=$ 1.17) than those for whom this was not done $(M=27.35, S D=1.67): t$ $(128)=2.99, \mathrm{p}=0.003$. On average, the group that was swabbed stayed in hospital for $\mathbf{6}$ days less, following their operation.

The records included another potential 'start' date, the date of diagnosis. A t-test was also performed to determine whether there was a difference in hospital stays from the date of diagnosis, between those patients who had received a culture swab and those who had not. It was found that hospital days were significantly lower in patients for whom culture swabs were completed $(\mathrm{M}=13.44, \mathrm{SD}=1.08)$ than those for whom this was not done $(\mathrm{M}=19.38, \mathrm{SD}=1.51): \mathrm{t}(129)=3.31 \mathrm{p}=$ $0, .0016$. On average, the group that was swabbed stayed in hospital for $\mathbf{5}$ days less, after suspected diagnosis of sepsis. Figure 3.4 shows the overall pattern of patient stays since the project commenced.

\footnotetext{
${ }^{6} \mathrm{We}$ do not have statistics from the pre-intervention phase on this. Women were tracked, where possible post-discharge by telephone. Tracking proved difficult in around $20 \%$ cases with some women not responding to calls. However, re-admission would have been evident through ward-based reporting.
} 


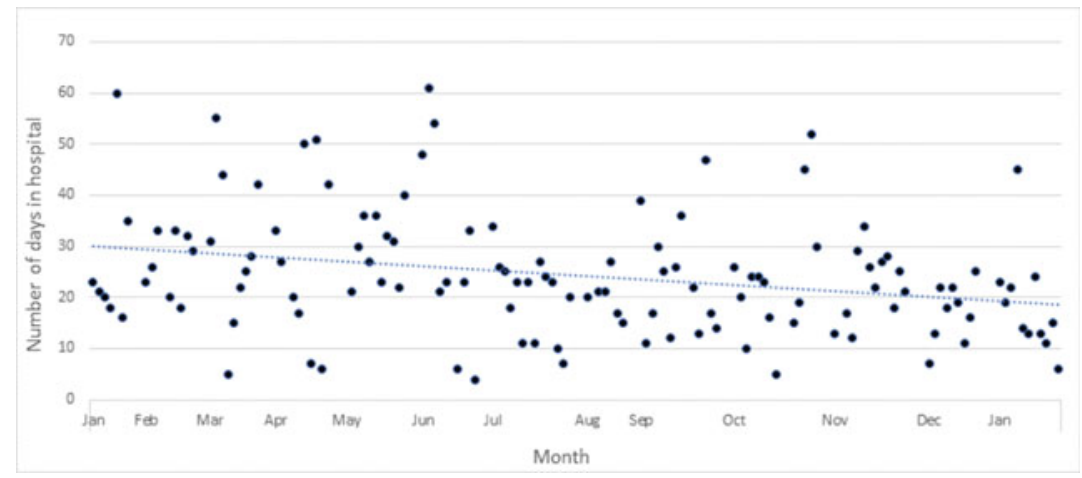

Fig. 3.4 Length of stay based on date of operation (Source Patient Records PNG Ward FPRRH [January 2019-January 2020])

As noted above, these reductions in patient stays will be associated with multiplier reductions in the volume of attendants staying in and around the wards.

\section{IMPACTS ON HOSPITAL BUDGETS}

Finally, and inevitably, extended stays represent a major cost to the hospital in terms of infrastructure, staffing and consumables. The effect of these improvements has been welcomed by the Senior Administrator who comments on the efficiencies this has generated:

When sepsis is managed then the resources used to manage these patients reduce drastically. By reducing long stays which brings about savings you have contributed to the hospital budget with real term savings. That's an area for me now to look into. We should be able to see in real terms how much savings we have been able to make.

Allegranzi et al. (2018) noted the paucity of studies identifying in any systematic way, the costs involved in managing health care associated infections and the cost-savings associated with effective interventions. The MSI did not set out to undertake detailed cost-benefit analysis. Nevertheless, as the project has evolved, and cost savings were remarked upon by respondents and senior managers, we started to explore the potential for integrating cost-benefit analysis in future work. This would provide 
critical impetus for sustainability and future investment. Our findings confirm Weber et al.'s assertion that strengthening WASH services in Togo was cost-minimal. Although key barriers cited by Weber's respondents referred to costs this was more a matter of institutional priorities than actual costs which are in reality very low and, importantly, could be managed within existing budgets, 'without significant external financial or material support' (2018: 1). The authors also recommend greater attention to cost-effectiveness and budget expenditures in future research (p. 64).

\section{PATIENT ExPeriences}

It is difficult to gauge patients' perceptions of the project's impact mainly because most will have nothing to compare their experience to. However, several midwives reported positive patient feedback:

The mothers have noticed the project because we had a mother who had sepsis. It was her second child and she had delivered in Buhinga before but that time she didn't have sepsis. She said, 'hey you people this ward has changed'. In the first pregnancy she delayed on the ward. This time she got sepsis, so we moved her from post-natal to gynae and she said 'hey you nurses why have you changed? You have changed me to gynae ward - now what is taking place?' We told her be calm and after a few days we worked on her and she went home. She said the hospital has changed! Nowadays they know.

The in-charge also noted genuine changes in the attitudes of staff towards their patients:

Some used to treat a death of a mother as if it were the death of a chicken. Now they see our patients as their sisters or mothers and care for them. Before some staff were hard to deal with; they barked at patients. The care in this ward is critical care and a woman could lose her life in one minute. We need the best and most caring staff.

The following chapter examines some of the constituent interventions and impacts that, together, contributed to the improvements described above. We start with a discussion of infection prevention and control. The 'control' dimension and the focus on wound management merges seamlessly into a discussion about antimicrobial surveillance and stewardship. 


\section{RefERENCES}

Allegranzi, B., Nejad, S. B., Combescure, C., Graafmans, W., Donaldson, L., \& Pittet, D. (2011). Burden of endemic health-care-associated infection in developing countries: Systematic review and meta-analysis. Lancet, 377, 228241.

Allegranzi, B., Aiken, A. M., Zeynep, K. N., Nthumba, P., Barasa, J., Okumu, G., et al. (2018, May). A multimodal infection control and patient safety intervention to reduce surgical site infections in Africa: A multicentre, beforeafter, cohort study. The Lancet Infectious Diseases, 18(5), 507-515. https:// doi.org/10.1016/S1473-3099(18)30107-5.

Filippi, V., Ronsmans, C., Gohou, V., Goufodji, S., Lardi, M., Sahel, A., et al. (2005). Maternal wards or emergency obstetric rooms? Incidence of near-miss events in African hospitals. Acta Obstet Gynecol Scand, 84, 11-16.

Hoonhout, L., de Bruijne, M. C., Wagner, C., Zegers, M., Waaijman, R., Spreeuwenberg, P., et al. (2009). Direct medical costs of adverse events in Dutch hospitals. BMC Health Services Research, 9, 27. https://doi.org/10. 1186/1472-6963-9-27.

Kongnyuy, E. J., Mlava, G., \& van den Broek, N. (2009). Facility-based maternal death review in three districts in the central region of Malawi: An analysis of causes and characteristics of maternal deaths. Women's Health Issues, 19(1), $14-20$.

Ngonzi, J., Tornes, Y. F., Mukasa, P. K., Salongo, W., Kabakyenga, J., Sezalio, M., et al. (2016). Puerperal Sepsis, the leading cause of maternal deaths at a Tertiary University Teaching Hospital in Uganda. BMC Pregnancy and Childbirth, 16, 207.

Seni, J., Najjuka, C. F., Kateete, D. P., Makobore, P., Joloba, M. L., Kajumbula, H., et al. (2013). Antimicrobial resistance in hospitalised surgical patients: A silently emerging public health concern in Uganda. BMC Research Notes, 6, 298.

Slawomirski, L., Auraaen, A., \& Klazinga, N. (2017). The economics of patient safety. OECD.

Weber, N., Patrick, M., Hayter, A., Martinson, A. L., \& Gelting, R. (2018). A conceptual evaluation framework for the water and sanitation for health facility improvement tool (WASH FIT). Journal of Water, Sanitation and Hygiene for Development, 9(2). https://doi.org/10.2166/washdev.2019.090.

Zulfiuqar, B., Salam, A., Firoz, M., Fatima, H., \& Aziz, S. (2013). Effects of inflow of inpatients attendants at a tertiary care hospital - A study at civil hospital Karachi. Journal of Pakistan Medical Association, 63(1), 143-147. 
Open Access This chapter is licensed under the terms of the Creative Commons Attribution 4.0 International License (http://creativecommons.org/licenses/ by $/ 4.0 /$ ), which permits use, sharing, adaptation, distribution and reproduction in any medium or format, as long as you give appropriate credit to the original author(s) and the source, provide a link to the Creative Commons license and indicate if changes were made.

The images or other third party material in this chapter are included in the chapter's Creative Commons license, unless indicated otherwise in a credit line to the material. If material is not included in the chapter's Creative Commons license and your intended use is not permitted by statutory regulation or exceeds the permitted use, you will need to obtain permission directly from the copyright holder.

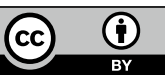

